\title{
On Joint ML Detection and Decoding for Linear Block Codes
}

\author{
Haris Vikalo and Babak Hassibi ${ }^{1}$ \\ Department of Electrical Engineering \\ California Institute of Technology \\ 1200 E. California Blvd., Pasadena, CA 91125 \\ $\{$ hvikalo, hassibi\}@systems. caltech.edu
}

Abstract - We consider joint maximum-likelihood (ML) detection and decoding in multiple-input multiple-output (MIMO) systems. The information data is encoded by a linear block error-correcting code and then transmitted across the MIMO channel in AWGN. Geometrically, the transmitted symbol is a point in a high-dimensional lattice. The received symbol is the lattice point perturbed by an additive noise. The joint detection and decoding problem is equivalent to the search for the closest lattice point that is an admissible codeword. We propose an algorithm which performs a search constrained by a sphere centered at the observed point. The radius of the sphere is determined according to the statistics of the noise. Thus the computational complexity of the algorithm is a random variable. We quantify it by means of its first moment which, for binary codes, we find analytically. The expected complexity of the proposed algorithm is polynomial in the length of the uncoded information word over a wide range of SNRs.

\section{SUMMARY}

We consider the model

$$
\mathbf{x}=H \mathbf{s}+\mathbf{v},
$$

where $H \in \mathcal{R}^{n \times m}$ has i.i.d. $\mathcal{N}(0,1)$ entries and $\mathbf{v} \in \mathcal{R}^{n \times 1}$ has i.i.d. $\mathcal{N}\left(0, \sigma^{2}\right)$ entries. The $m$-dimensional vector $s$ is obtained by modulating a coded word $\mathrm{c}$ onto an $L$-PAM constellation. The $m$-dimensional vector $c$ is obtained by encoding a $p$-dimensional information vector $\mathbf{b}$ by a linear block $p / m$-rate code. The code is defined by an $m \times p$ generator matrix $\mathbf{G}$ (i.e., $\mathbf{c}=\mathbf{G} \cdot \mathbf{b}$ ). The entries in $\mathbf{c}, \mathbf{b}$, and $\mathbf{G}$, are assumed to be elements from a Galois field $G F(L)$ and the operations involving them are modulo- $L$.

The ML decoding problem can be stated as

$$
\min _{\mathbf{b} \in G F(L)^{p}}\|\mathbf{x}-H \mathbf{s}\|^{2}
$$

A straightforward (but intractable) way of obtaining the solution to the ML decoding problem is by means of an exhaustive search. Motivated by [1], we propose an efficient alternative: an algorithm that solves (2) by searching only over those points in the lattice $H \mathbf{s}$ that belong to a hypersphere around the observed point $\mathbf{x}$ and are admissible codewords.

To this end, we first require some pre-processing of the matrix G. In particular, we transform $\mathbf{G}$ into an approximately upper-triangular form $G$ shown below:

\footnotetext{
${ }^{1}$ This work was supported in part by the National Science Foundation under grant no. CCR-0133818, by the Office of Naval Research under grant no. N00014-02-1-0578, and by Caltech's Lee Center for Advanced Networking.
}

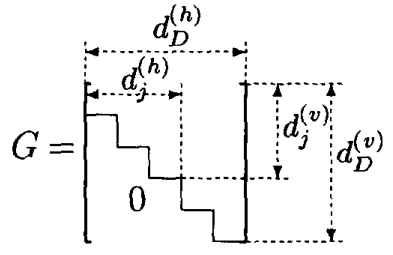

Operations that are allowed in the process of transforming $G$ into $G$ are permutations and additions of the rows (i.e., operations that preserve properties of the code).

As in [1], to solve (2) we search for points inside a sphere of appropriately chosen radius $r$. This is equivalent to solving

$$
\sum_{j=1}^{D}\left\|R_{j j}\left(\mathbf{s}_{j}-\hat{\mathbf{s}}_{j}\right)+\sum_{k=j+1}^{D} R_{j k}\left(\mathbf{s}_{k}-\hat{\mathbf{s}}_{k}\right)\right\|^{2} \leq r^{2},
$$

where $R_{j k}=R\left(d_{j-1}^{(h)}+1: d_{j}^{(h)} ; d_{j-1}^{(v)}+1: d_{j}^{(v)}\right), R$ is a matrix in $Q R$ factorization of $H$ (i.e., $H=Q R$ ), and where

$$
\mathbf{s}_{j}=\left[s_{d_{j-1}^{(v)}} \cdots s_{d_{j}^{(v)}}\right]^{T} \quad \hat{\mathbf{s}}_{j}=\left[\hat{s}_{d_{j-1}^{(v)}} \ldots \hat{s}_{d_{j}^{(v)}}\right]^{T}
$$

$j=1,2, \ldots, D, j<k \leq D$

As an example, consider rate- $1 / 2$ Golay 24 code. Comparison of BER performance of the algorithm with that of hard ML detection followed by ML decoding is shown in Figure 2. The same figure shows expected complexity exponent $e_{c}=\log _{m}$ (\# flops per decoded word) of the algorithm. The expected complexity (which we obtain analytically) is polynomial (roughly $c_{e} \approx 4$ ) over the considered range of SNRs.
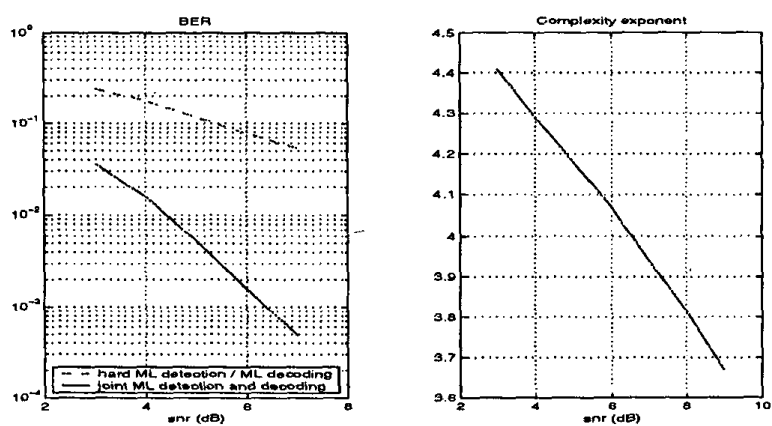

Figure 1: BER performance and complexity of the algorithm for joint detection and decoding for Golay 24 code.

\section{REFERENCES}

[1] U. Fincke and M. Pohst, "Improved methods for calculating vectors of short length in a lattice, including a complexity analysis," Mathematics of Computation, vol. 44, pp. 463-471, April 1985. 